\title{
Lithium insertion studies on boron-doped diamond as a possible anode material for lithium batteries
}

\author{
A. Y. M. T. Christy • Kee Suk Nahm • Yun Ju Hwang • \\ E. K. Suh • M. Anbu Kulandainathan - T. Premkumar • \\ A. Manuel Stephan
}

Published online: 19 February 2008

(C) Springer-Verlag 2008

\section{Erratum to: Ionics}

\section{DOI 10.1007/s11581-007-0164-1}

Unfortunately, Tables 1 and 2 were not printed. The tables were given here.

Table 1 The insertion and deinsertion capacities of boron-doped diamond electrode

\begin{tabular}{lll}
\hline Cycle number & $\begin{array}{l}\text { De-insertion capacity } \\
\mathrm{mAh} \mathrm{g}^{-1}\end{array}$ & $\begin{array}{l}\text { Insertion capacity } \\
\mathrm{mAh} \mathrm{g}^{-1}\end{array}$ \\
\hline 1 & 0.0061 & 8.1851 \\
2 & 2.0909 & 2.1333 \\
3 & 0.9017 & 1.0197 \\
4 & 0.7656 & 0.8533 \\
5 & 0.7625 & 0.8533 \\
6 & 0.7232 & 0.8049 \\
7 & 0.7111 & 0.7958 \\
\hline
\end{tabular}

Table 2 The insertion and deinsertion capacities of boron-doped diamond with nanotin as additive

\begin{tabular}{lll}
\hline Cycle number & $\begin{array}{l}\text { De-insertion capacity } \\
\mathrm{mAh} \mathrm{g}^{-1}\end{array}$ & $\begin{array}{l}\text { Insertion capacity } \\
\mathrm{mAh} \mathrm{g}^{-1}\end{array}$ \\
\hline 1 & 68.5 & 134.5 \\
2 & 128.8 & 131.3 \\
3 & 122.3 & 124.9 \\
\hline
\end{tabular}

The online version of the original article can be found at http://dx.doi.org/10.1007/s11581-007-0164-1.

A. Y. M. T. Christy · K. S. Nahm • Y. J. Hwang • E. K. Suh ( $\bowtie)$

School of Semiconductor and Chemical Engineering,

Chonbuk National University,

Chonju 561- 756, South Korea

e-mail: eksuh@chonbuk.ac.kr

M. A. Kulandainathan - T. Premkumar · A. M. Stephan

Central Electrochemical Research Institute,

Karaikudi 630 006, India 